\title{
Acoustic Signal Based Fault Detection of Motorcycles Using Slope Vector of the Estimated Pseudospectrum
}

\author{
Basavaraj S. Anami ${ }^{1}$ and Veerappa B. Pagi ${ }^{2}$ \\ ${ }^{1}$ KLE Institute of Technology, Hubli-580 030, Karnataka State, India \\ ${ }^{2}$ Basaveshwar Engineering College, Bagalkot - 587 102, Karnataka State, India \\ anami_basu@hotmail.com,veereshpagi@gmail.com
}

\begin{abstract}
The automobile service experts often assess the health condition of the motorcycles based on the sound produced by taking test rides. To be effective, this process of fault diagnosis needs to be automated. The purpose of this paper is to present a method for fault detection of motorcycles that employs the slopes of the pseudospectral segments as features. Further, the estimated pseudospectrum of a sound signal is divided into eight segments, and the slope of each segment is computed. Artificial neural network (ANN) classifier is used for classification. The experimental results show that the proposed method achieves satisfactory results with an average accuracy of $78 \%$ for healthy motorcycles and $89 \%$ for faulty motorcycles. The study can be extended to locate the faults in subsystems of vehicles. The proposed work finds applications in allied areas such as fault diagnosis of machinery, musical instruments, electronic gadgets etc.
\end{abstract}

Keywords: Vehicle fault diagnosis; acoustical signal processing; pseudospectrum; neural network classifier

\section{Introduction}

Motorcycles are the most favorable travel companions in India. They account for major Indian automobile market share with nearly $77 \%$ of the total vehicle sales. Society of Indian Automobile Manufacturers (SIAM) has forecast the two-wheeler segment to register a growth of $6 \%-8 \%$ in $2013-14$ [1]. The sound patterns generated by the vehicles convey information necessary for fault diagnosis. People depend on the service station experts for the repair and maintenance of vehicles. Automated fault diagnosis systems are useful in assessing the condition of vehicles in remote places, places of scarce expertise and in service stations for preliminary analysis of the faults.

Non-speech sound signal processing is a challenging task due to lack of sound alphabet. Vehicle classification based on sound is a non-speech sound processing application that poses difficulty in terms of the analysis of the sound patterns. The main factors that affect the variations in sound are mainly the variations in speed, health condition, road condition, surrounding environment etc. The sound of a moving vehicle helps the rider to know the status of the vehicle, whereas the sound of a stationary vehicle with running engine helps the mechanic to assess its working condition.

Most of the faults leave a trace of the clue before they turn severe. If the problem is not attended in time it may lead to abrupt failure and leads to a probable accident. The problem of motorcycle fault detection is significant since it helps the rider to know the condition of the motorcycle, before the problem becomes severe. In this work, the slopes of the pseudospectral 
segments are used as features and ANN as classifier. The work leaves scope for further investigation of faults from various subsystems of motorcycles.

The novelty of the work lies in using the estimated pseudospectrum of the sound signals for computing the features. The reported works use time-domain, spectral and time-frequency analysis for feature extraction of acoustic signals of vehicles. But the estimated pseudospectra are unique for sound signals of healthy and faulty motorcycles. Hence the pseudospectrum is divided and analyzed in eight regions to form a slope vector of length eight. The computed slope vector is used as a feature vector input for classification. The approach is novel since there is no reported work exploiting the variations in pseudospectral curve. The work helps in traffic census of the vehicles, checking the unlawful mixture of fuel, automatic acoustic surveillance and the like.

\section{Background}

Sound signal processing techniques provide an ideal means for real time analysis of classifying the vehicles into engine fault diagnosis [2, 3, 4, and 5], gearbox fault diagnosis [6, 7 and 8], bike and scooter [13]. The state-of-the-art related to the proposed study is analyzed and the studied literature is organized into three components: engine fault diagnosis, gearbox fault diagnosis, and other applications.

[2] Present the fault detection system for motorcycles based on acoustic signals. The approach employs the 1D central contour moment and their invariants of wavelet subbands and DTW classifier. [3] Discuss the mechanisms of engine front noise generation and the corresponding countermeasures of a diesel engine using sound intensity method. [4] use continuous wavelet transform and ANN to develop a mechanical fault diagnosis system for a scooter engine platform. [5] Use empirical mode decomposition (EMD) and wavelet packet backpropagation neural network for engine fault diagnosis.

[6] Present a methodology for fault diagnosis of Massey Ferguson gearbox using root mean square (RMS) and power spectral density (PSD). [7] Propose a system for detection of the vibration signals of a gearbox with early fatigue tooth crack employing adaptive wavelet filter. [8] Provide an approach for the classification of the working condition of gear. The approach decomposes the vibration signals into a finite number of intrinsic mode functions (IMFs) and then establishes the autoregressive (AR) model of each IMF component and finally generates the corresponding autoregressive parameters. The work regards the autoregressive parameters and the variance of remnant is regarded as the fault characteristic vectors and uses them as input parameters of SVM classifier.

Condition monitoring application presented by [9] uses features such as magnitude of the signal, natural logarithm of the magnitude and Mel-frequency cepstral coefficients (MFCC). The features are used as input to various pattern classifiers. [10] compare the fault detection alternatives for induction machines according to the information required for the diagnosis, the number and relevance of the faults that can be detected, the speed to anticipate a fault and the accuracy in the diagnosis. [11] Relate a multi-resolution wavelet analysis with a neural network for the fault analysis of industrial robots. [12] Apply wavelet analysis of accelerometric signals for detection of wheelflat faults in railway. [13] Employ simple time domain and frequency domain features as inputs for neural network model for classifying motorcycles into bikes and scooters, based on acoustic signatures. [14] Demonstrates a structure for monitoring the state of a turbocharger and supervising the air pressure in vehicle wheels. The structure involves fuzzy inference mechanism based on neural units. The approach combines both the adaptive feature of neural networks and the transparency of 
fuzzy systems. [15] Illustrates a denoising method based on the wavelet technique for feature sound extraction for diagnosis of machines.

Wavelet-based approaches yield better results, because of the better time-frequency resolution. IMF-AR based approach with SVM classifier for fault diagnosis of gears which results in 100\% classification accuracy [7]. An approach that employs time-domain and frequency-domain features with ANN yields an accuracy of $72 \%$ while classifying the motorcycles into bikes and scooters [13]. Continuous wavelet transform (CWT) can be used for machine fault detection based on sound. The sound purified with CWT can be used to extract the feature sound from the noisy signal. The extracted feature sound can help the operators to diagnose the machine correctly, even if the machine sound has a low SNR [15]. In the similar fashion, the denoised signal aids for easier fault diagnosis of vehicles. Since the existing approaches employ different databases recorded in different environments and use denoising, it is difficult to compare the findings of our work with the reported works. Table 1 summarizes the wavelet-based works for fault diagnosis and vehicle classification.

Table 1. A Comparative Study of Wavelet-based Works

\begin{tabular}{|c|l|c|l|l|l|l|}
\hline $\begin{array}{l}\text { Sl. } \\
\text { No. }\end{array}$ & $\begin{array}{l}\text { Type of } \\
\text { work }\end{array}$ & Article & Features & Classifier & $\begin{array}{l}\text { No. of } \\
\text { Classe }\end{array}$ & Accuracy (\%) \\
\hline 1. & AC & {$[16]$} & $\begin{array}{l}\text { HLA, DWT, } \\
\text { STFT, PCA }\end{array}$ & $\begin{array}{l}\text { k-NN, } \\
\text { MPP }\end{array}$ & 4 & $\begin{array}{l}\text { k-NN: 85 } \\
\text { MPP: 88 }\end{array}$ \\
\hline 2. & AC & {$[17]$} & CWT & MLNN & 6 & $95-100$ \\
\hline 3. & FD & {$[18]$} & DB-20 wavelets & ANN & 2 & $70-100$ \\
\hline 4. & FD & {$[11]$} & DWT & - & 6 & $45-100$ \\
\hline 5. & FM & {$[4]$} & CWT & ANN & 5 & 95 \\
\hline 6. & FM & {$[2]$} & DB4 \& moments & DTW & 2 & $81-100$ \\
\hline 7. & FM & {$[12]$} & DWT & FFNN & 2 & $94-100$ \\
\hline 8. & VC & {$[19]$} & DWT & $\begin{array}{l}\text { MLNN, } \\
\text { PNN }\end{array}$ & 4 & $\begin{array}{l}\text { MLNN: 71 } \\
\text { PNN: 73 }\end{array}$ \\
\hline 9. & VC & {$[20]$} & DWT & MPP & 2 & 98 \\
\hline
\end{tabular}

Legend: AC - Audio classification; ANN - Artificial neural network; CWT Continuous wavelet transform; DBn - Daubechies wavelet of the order $n$; DWT Discrete wavelet transform; FD - Fault diagnosis; FFNN- feedforward neural network; FM - Fault diagnosis of vehicle; HLA - Harmonic line association; $k$-NN - $k$-Nearest neighbor classifier; MLNN - Multilayer neural network; MPP - Minimum distance approach; PCA - Principal component analysis; PNN - Probabilistic neural network; STFT - Short time Fourier Transform; VC - Vehicle classification

From the literature survey, it is evident that reasonable amount of research is reported for various applications of sound signal analysis using various techniques including wavelets. Since no work is reported on fault detection of motorcycles using pseudospectrum, the study is taken up.

The remainder of the paper is organized into 3 sections. The proposed methodology of the work is discussed in Section 3; the experimental results are discussed in Section 4. Finally, the Section 5 concludes the work. 


\section{Proposed Methodology}

The proposed methodology for fault detection of motorcycles based on sound employs the MUSIC algorithm [23] to estimate the pseudospectrum. The slopes of the first five segments of the estimated pseudospectrum are used as features. The computed features are input to the ANN classifier for classification into healthy and faulty. The overview of the proposed methodology is depicted in Figure 1. It comprises four stages namely, sound signal acquisition, segmentation, feature extraction and classification. Each of these stages is explained in the subsections to follow.

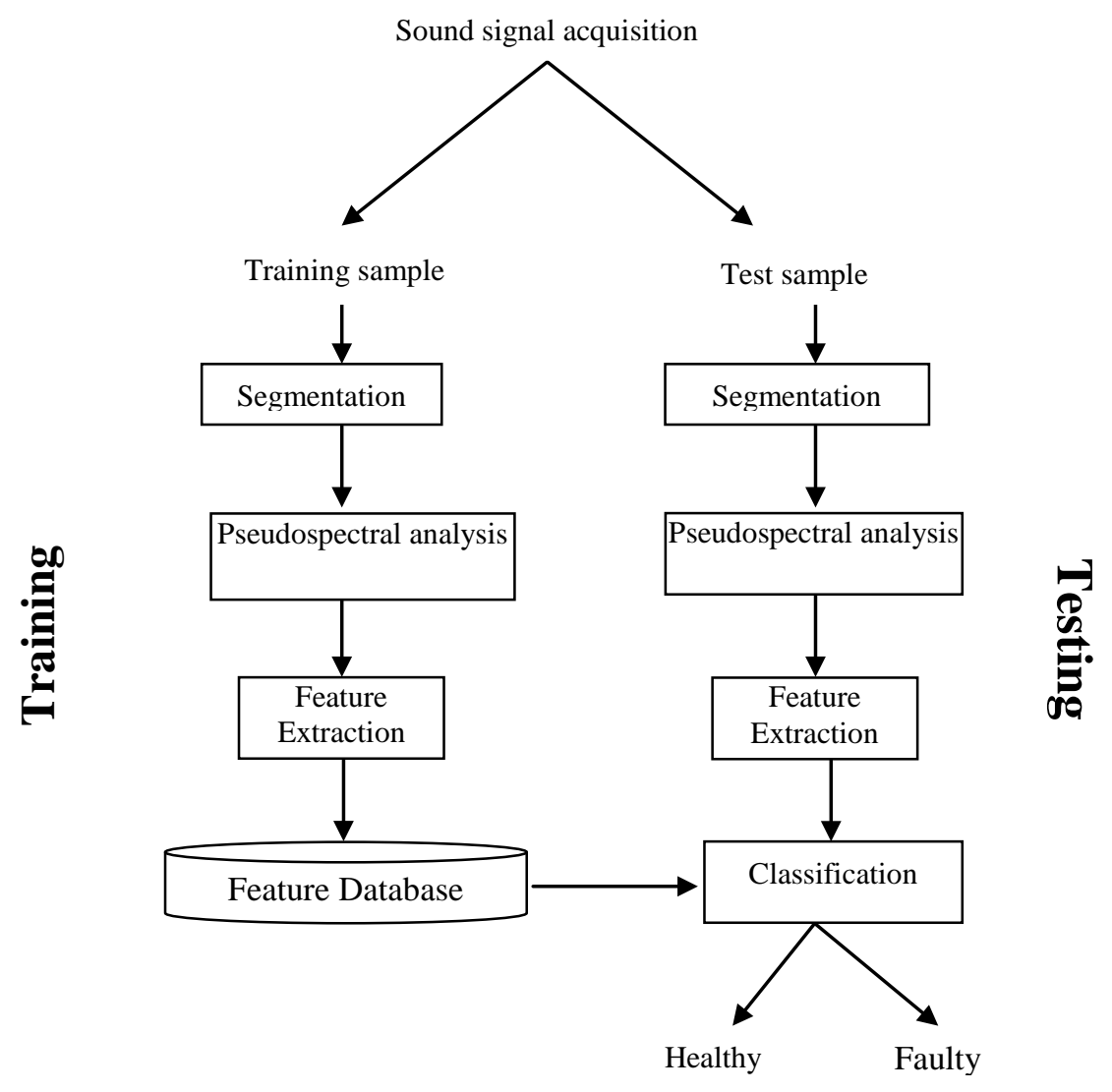

Figure 1. Block Diagram of the Proposed Method

\subsection{Recording of Sound Samples}

Recording is carried out under the supervision of expert mechanic in authorized service stations. Each sound sample is recorded using Sony ICD-PX720 digital voice recorder. While recording the sound signals the motorcycle is held in idling state. The signals are recorded at sampling frequency of $44.1 \mathrm{kHz}$ with 16 bit quantization.

The automotive guidelines recommend the sampling frequency of $9 \mathrm{kHz}$ to $30 \mathrm{kHz}$ as ideal for recording. However, this range of frequency is suitable only for recording in anechoic chamber with ideal setup. Since the objective is to work on real-world signals, the sampling frequency of $44.1 \mathrm{kHz}$ is used. It helps in capturing the signals sensitive to minor variations. Other factors influencing the classification are the variations in models chosen, the age of the vehicles, maintenance of the vehicles, denoising etc. Noise produced by surrounding objects also affects the quality of the recording. So we need a clear 5 meter radius from the centre 
where testing takes place. Since recording is carried out in service stations, wind effects are minimized.

The recording environment has influence on the classification performance Real time recording environment is always noisy with other sound sources such as human speech, sounds of other vehicles, noise of air-compressor and equipments. No preprocessing is carried out over the sound samples acquired with the intention of processing them in the real-world environment. Figure 2 depicts the environment maintained during the recording the motorcycle sounds. The recorder is held $500 \mathrm{~mm}$ from the centre line of the exhaust end, and at an angle of about $45^{\circ}$ measured from the centre line of the exhaust end. The $500 \mathrm{~mm}$ is critical, as an $80 \mathrm{~mm}$ error either way will result in around one decibel increase or decrease in sound level. The start of the engine and the throttle are controlled by the expert mechanic at the same time.

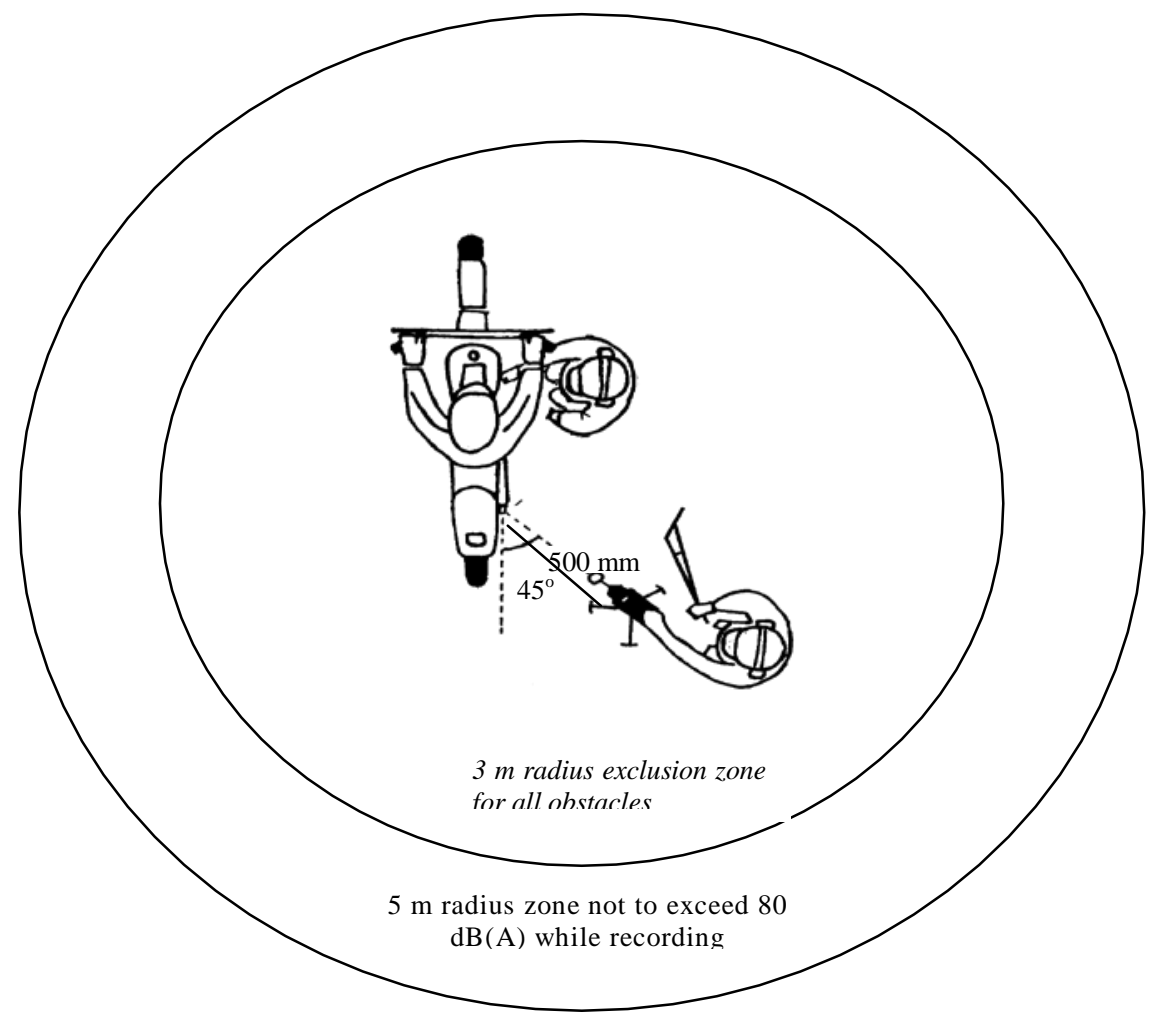

Figure 2. Recording Environment

\subsection{Segmentation of Sound Signals}

The acquired sound signals are segmented into samples of one-second duration each for uniformity in processing. The segment begins at the local maxima within $50 \mathrm{~ms}$ duration and runs for one second. The next segment begins at local maxima in the next 50 ms duration.

\subsection{Feature Extraction}

The features are extracted from the pseudospectral estimation of the signal. The entire pseudospectrum in the range of normalized frequencies is divided into eight regions. The slope of the spectrum in each region is computed, and results in eight-valued vector. The 
Subsections 3.3.1 and 3.3.2 discuss the pseudospectrum estimation and the slopes of the segments respectively.

3.3.1. Pseudospectrum Estimation: The pseudospectrum is calculated using estimates of the eigenvectors of a correlation matrix associated with the input data $\mathrm{x} .[\mathrm{S}, \mathrm{w}]=\operatorname{pmusic}(\mathrm{x}, \mathrm{p})$ implements the MUSIC (Multiple Signal Classification) algorithm and returns $\mathrm{S}$, the estimated spectrum of the input signal $\mathrm{x} . \mathrm{p}$ is the signal subspace dimension and $\mathrm{w}$ is a vector of normalized frequencies (in rad/sample) at which the spectrum is evaluated.

The MUSIC estimate is given by the Equation (1).

$$
P_{\text {music }}(f)=\frac{1}{e^{H}(f)\left(\sum_{k=p+1}^{N} v_{k} v_{k}^{H}\right) e(f)}=\frac{1}{\sum_{k=p+1}^{N}\left|v_{k}^{H} e(f)\right|^{2}}
$$

where $\mathrm{N}$ is the dimension of the eigenvectors and $v_{k}$ is the $\mathrm{k}^{\text {th }}$ eigenvector of the correlation matrix of the input signal. The integer $\mathrm{p}$ is the dimension of the signal subspace, so the eigenvectors $v_{k}$ used in the sum correspond to the smallest eigenvalues and also span the noise subspace. The vector $e(f)$ consists of complex exponentials, so the inner product $v_{k}^{H} e(f)$ amounts to a Fourier transform. In the eigenvector method, the summation is weighted by the eigenvalues $\lambda_{k}$ of the correlation matrix is as given in Equation (2).

$$
P_{e v}(f)=\frac{1}{\left(\sum_{k=p+1}^{N}\left|v_{k}^{H} e(f)\right|^{2}\right) / \lambda_{k}}
$$

Figure 3 shows the average pseudospectra of sound signals of healthy and faulty motorcycles.

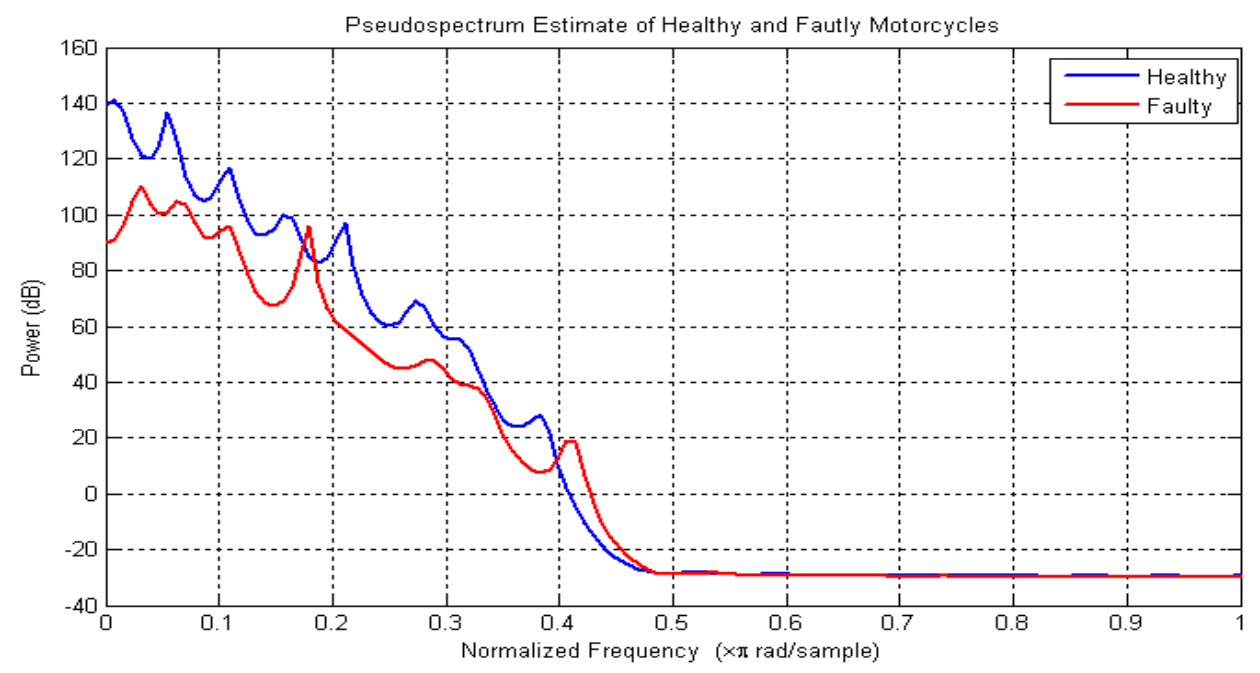

Figure 3. Spectra of Sound Signatures of Healthy and Faulty Motorcycles 
The spectral peaks decrease monotonically for healthy vehicles and no irregular variations are found in their spectra. But, in case of spectra of faulty motorcycles, the degraded harmonicity, non-monotonous decrease in spectral peaks and spurious peaks at higher frequencies, are observed. The overall power appears to be the same but the variations in spectrum can be observed for the normalized frequency below $0.45 \times \pi \mathrm{rad} / \mathrm{samples}$.

3.3.2. Slope of a Spectral Region: The spectral regions are formed in the normalized frequency domain. The computed pseudospectral estimate has 129 values. The curvature that runs in the said region is considered for computing the slope. The slope of the pseudospectrum is computed over the partitioned regions. First eight regions are considered for feature calculation. Figure 4 depicts the computation of slope of spectral region. The slope of the spectral region is computed by considering the maximum and minimum values of the estimated pseudospectral values in each region. The slope of each region is computed using Equation (3).

$$
\text { Slope }=20 \log 10\left(\frac{\max (S)-\min (S)}{\text { posmax }- \text { posmin }}\right)
$$

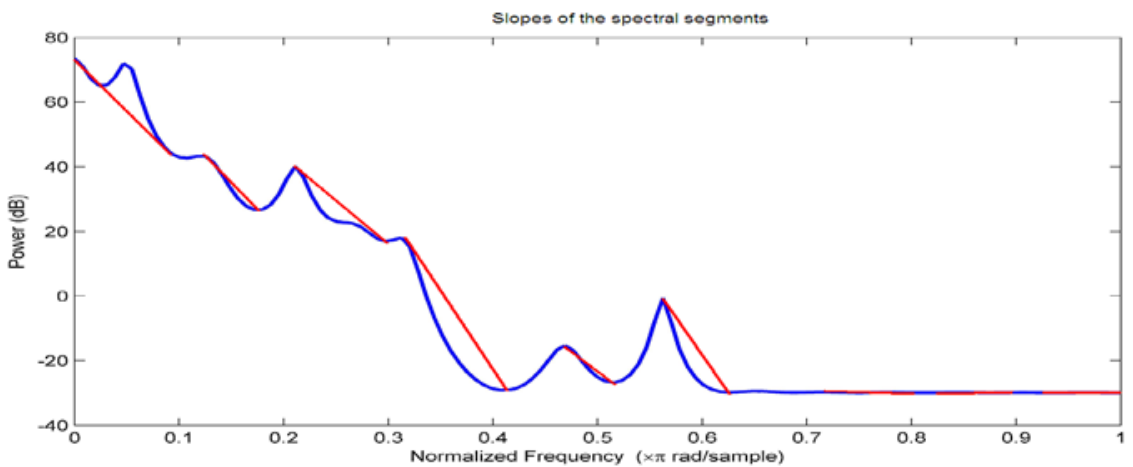

Figure 4. Spectral Regions Considered for Computing the Slope

Figure 5 shows the logarithmic plot of spectral signatures of different faults. From this figure it can be observed that different faults result in different spectral traces.

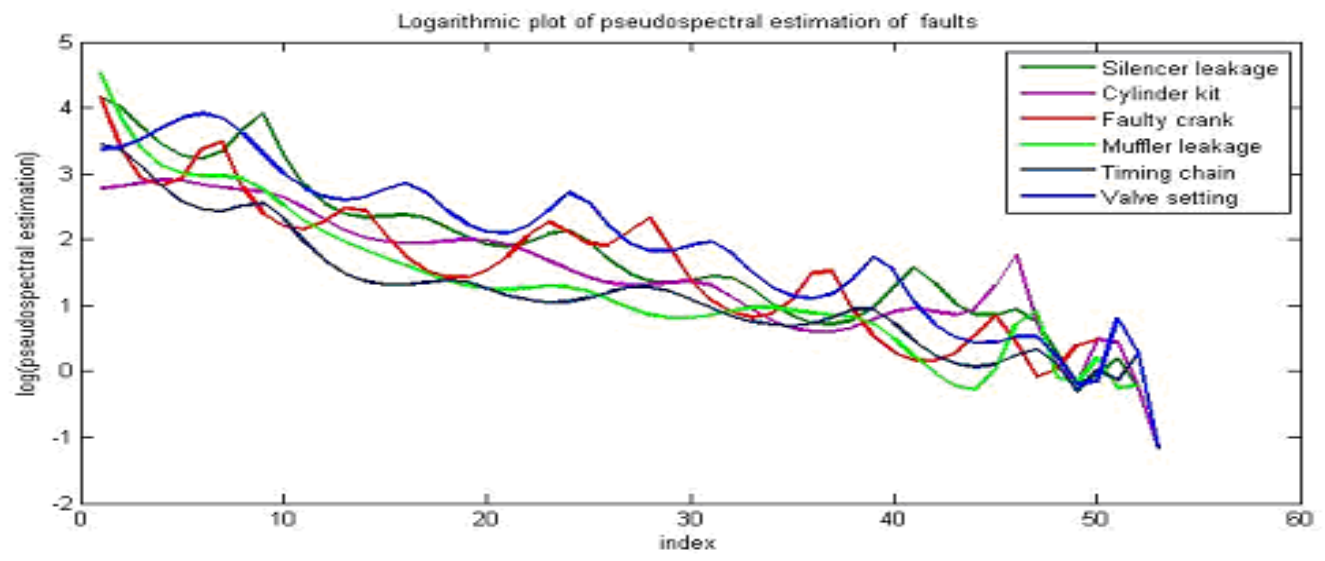

Figure 5. Logarithmic Plot of Pseudospectra of Faults 
Figures 6 to 9 depict the separability of the slopes of spectra in the first four regions, for healthy and faulty motorcycle sound samples. It can be clearly observed that the slopes differ for healthy and faulty motorcycles in these regions. Here the slope values are taken directly without converting to dBA.

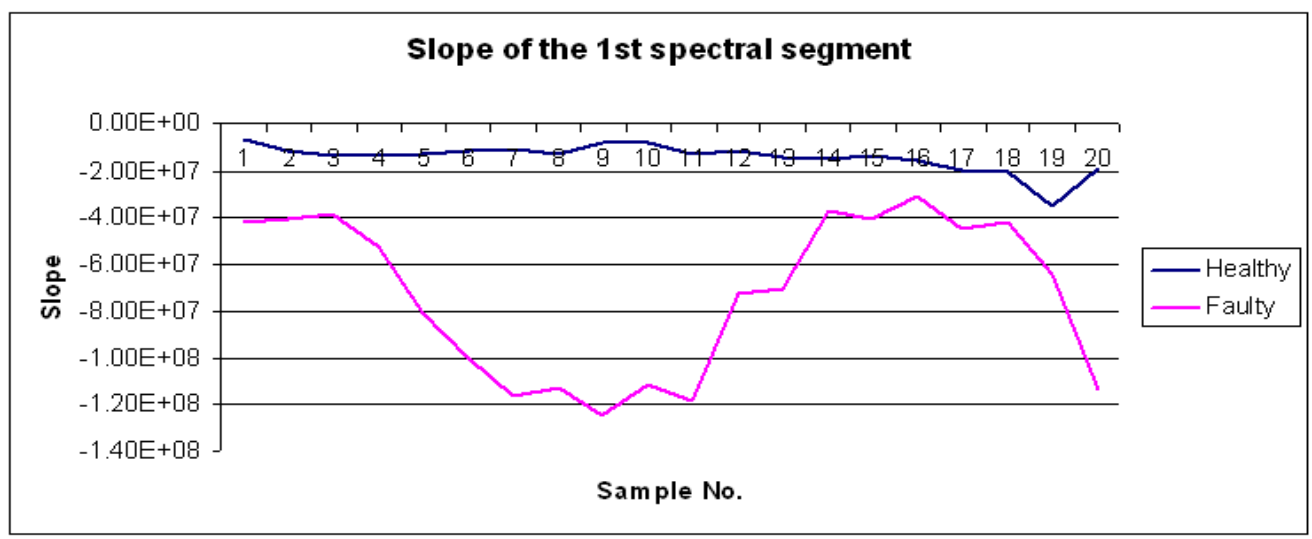

Figure 6. Slopes of the First Spectral Regions

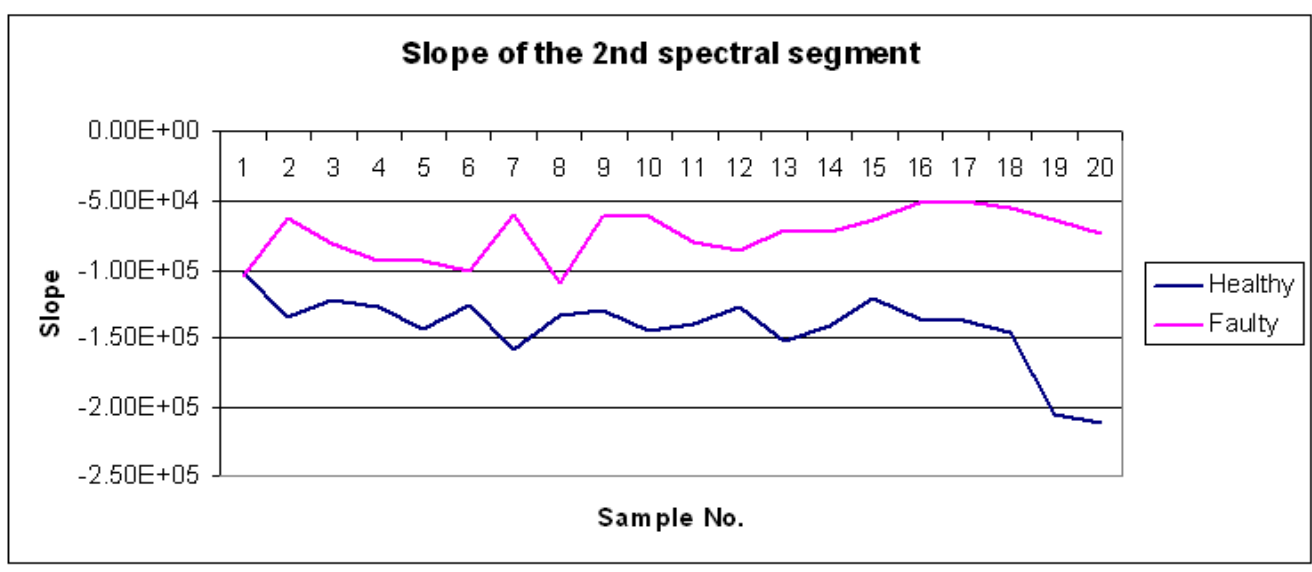

Figure 7. Slopes of the Second Spectral Regions

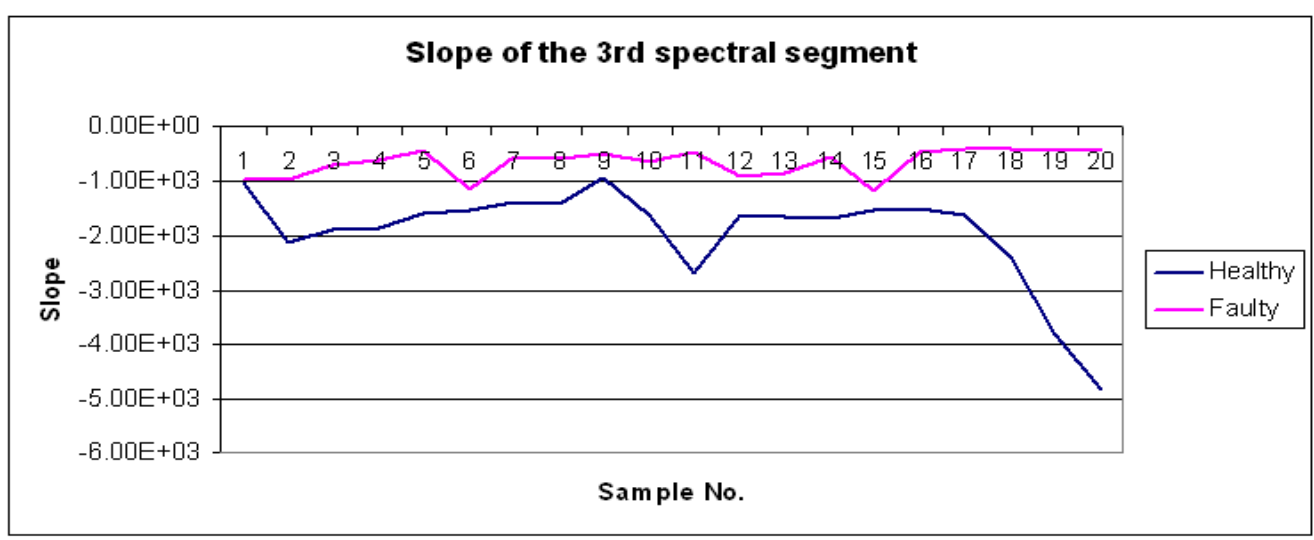

Figure 8. Slopes of the Third Spectral Regions 


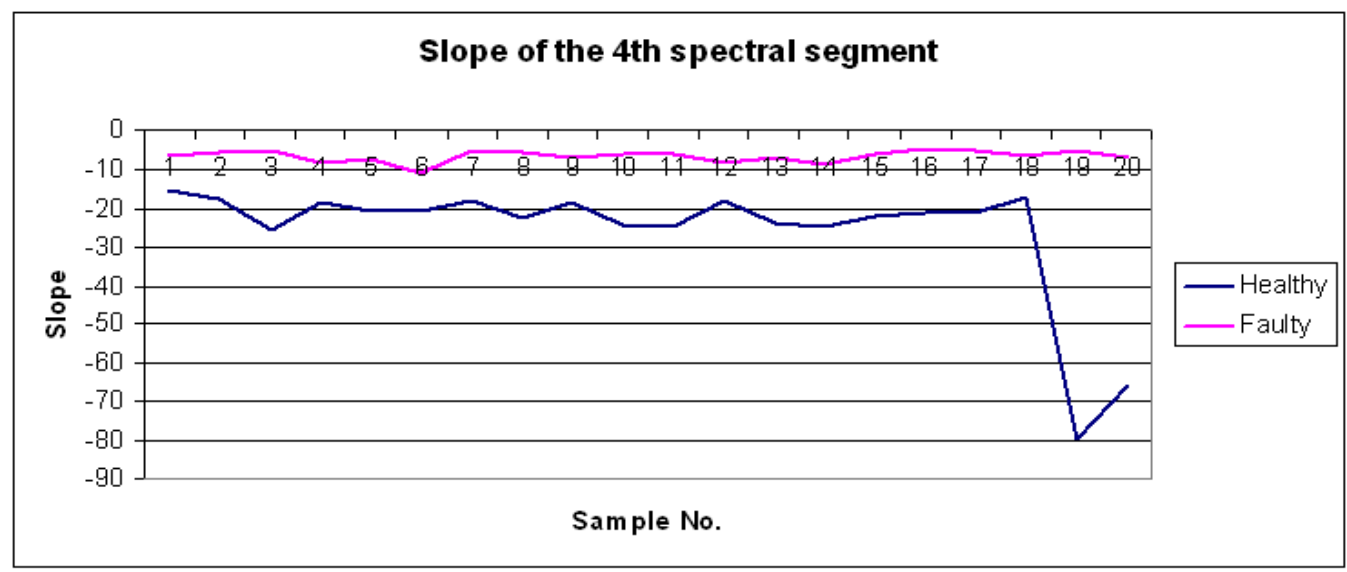

Figure 9. Slopes of the Fourth Spectral Regions

\subsubsection{ANN Classifier}

The computed feature values for the current problem exhibit slight variations. Hence the ANN classifier is chosen. Figure 10 shows the overview of the architecture of the ANN classifier. The eight features extracted based on the slopes of the pseudospectrum, are input to the neural network with four input nodes. The two output nodes correspond to the two-bit output vector indicating the health condition of the motorcycle. The hidden layer contains ten nodes. The neural network is trained using backpropagation-learning algorithm.

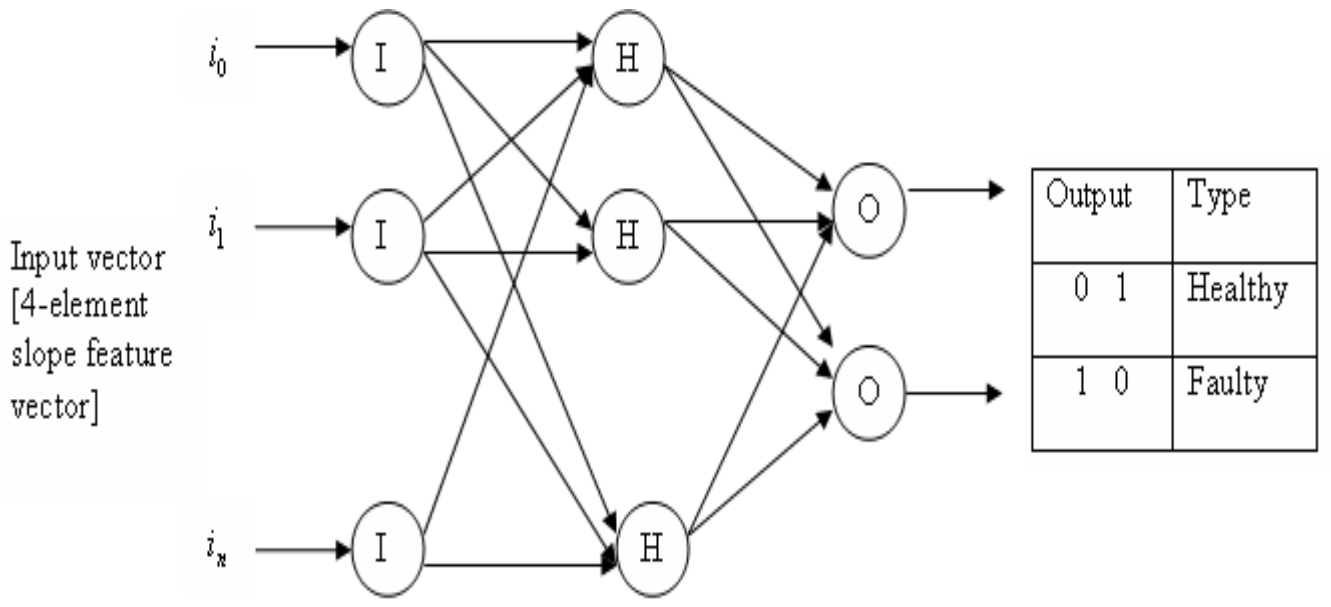

Figure 10. Architecture of the ANN

If the error is within the set limits, the training goal is said to be met. In such cases if testing is carried out on the same samples used for training, the classification accuracy will be appreciable. This usually happens for smaller training sample sets. If the training is terminated before the goal is met, the classification performance suffers. This is common for larger training sets.

The neural network is trained using backpropagation-learning algorithm. During testing, the stabilized weights are reloaded and the test vector is input. The optimal number of hidden layer neurons is chosen using the criterion given in Equation (4) discussed by [21, 22]: 


$$
n=C \sqrt{\frac{N}{d \log N}}
$$

where, $\mathrm{n}=$ number of hidden layer neurons, $\mathrm{C}=$ constant yielding optimal performance, $\mathrm{d}=$ number of features, and $\mathrm{N}=$ number of rows in the training sample matrix.

Validation set is used to design the ANN for optimal performance. The ANN exhibits optimal performance for minimum mean squared error (MSE). The MSE is plotted for varying number of hidden neurons from 3 to 15 . The sample sets with $70 \%$ of the samples used for training, $15 \%$ for validation and $15 \%$ for testing are used for computing the MSE. Figure 11 shows the validation MSE is used to decide the number of nodes in the hidden layer. Since the minimum MSE is observed when 10 nodes are used in the hidden layer, the hidden layer of the designed ANN contains 10 nodes.

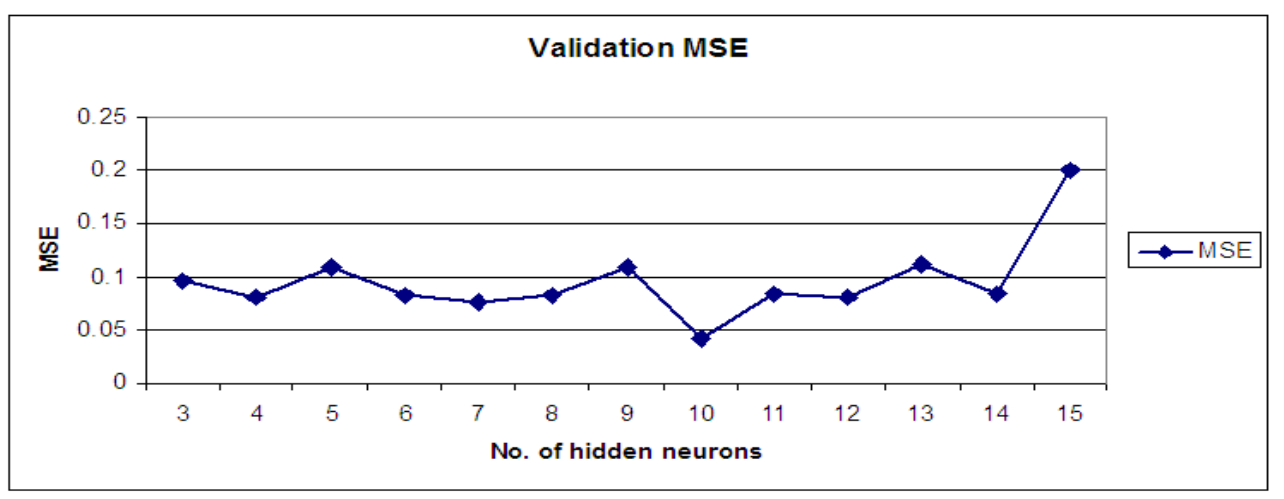

Figure 11. Validation MSE for Varying Number of Hidden Layer Neurons

\section{Results and Discussion}

Motorcycles of four popular Indian brands, namely Hero Honda (presently known as Hero Motocorp), Honda motors, TVS motors, and Bajaj motors Ltd., are considered. The database of motorcycle sound samples contains a total of 500 samples which includes 250 samples of healthy motorcycles and 250 samples of faulty motorcycles. The database is divided into three subsets: training, validation and testing with randomly chosen samples.

Table 2 shows the results of classification for various combinations of training, validation and testing sets with ANN classifier. The classification accuracies are nearly $100 \%$ for the used feature sets. The classification performance decreases with increase in the size of feature set. The performance of the built neural network is analyzed for different combinations of training, validation and testing sets. 
Table 2: Results of classification for ANN classifier

\begin{tabular}{|c|c|c|c|c|c|c|c|}
\hline \multicolumn{3}{|c|}{ No. of samples } & \multicolumn{5}{|c|}{$\begin{array}{c}\text { Combined results of classification for training, validation and test } \\
\text { samples (Total of } 500 \text { samples) }\end{array}$} \\
\hline Training & Validation & Testing & $\begin{array}{c}\text { True } \\
\text { Positive }\end{array}$ & $\begin{array}{c}\text { False } \\
\text { Negative }\end{array}$ & $\begin{array}{c}\text { True } \\
\text { Negative }\end{array}$ & $\begin{array}{c}\text { False } \\
\text { Positive }\end{array}$ & Accuracy \\
\hline 150 & 175 & 175 & 220 & 30 & 231 & 19 & 0.9020 \\
\hline 175 & 150 & 175 & 220 & 30 & 233 & 17 & 0.9060 \\
\hline 175 & 175 & 150 & 202 & 48 & 229 & 21 & 0.8620 \\
\hline 200 & 125 & 175 & 190 & 60 & 217 & 33 & 0.8140 \\
\hline 200 & 150 & 150 & 185 & 65 & 219 & 31 & 0.8080 \\
\hline 200 & 175 & 125 & 195 & 55 & 234 & 16 & 0.8580 \\
\hline 225 & 100 & 175 & 205 & 45 & 221 & 29 & 0.8520 \\
\hline 225 & 125 & 150 & 198 & 52 & 219 & 31 & 0.8340 \\
\hline 225 & 150 & 125 & 194 & 56 & 226 & 24 & 0.8400 \\
\hline 225 & 175 & 100 & 210 & 40 & 235 & 15 & 0.8900 \\
\hline 250 & 100 & 150 & 192 & 58 & 221 & 29 & 0.8260 \\
\hline 250 & 125 & 125 & 184 & 66 & 222 & 28 & 0.8120 \\
\hline 250 & 150 & 100 & 199 & 51 & 224 & 26 & 0.8460 \\
\hline 275 & 100 & 125 & 199 & 51 & 207 & 43 & 0.8120 \\
\hline 275 & 125 & 100 & 227 & 23 & 226 & 24 & 0.9060 \\
\hline 300 & 100 & 100 & 195 & 55 & 229 & 21 & 0.8480 \\
\hline
\end{tabular}

The minimum classification accuracies are $98 \%$ for faulty samples when the database has 50 samples of healthy and 50 samples of faulty. The classification accuracy degrades with increase in number of samples in the database. Finally, a database with 500 samples (250 samples of healthy and 250 samples of faulty) yields $68.4 \%$ and $82.8 \%$ respectively for healthy and faulty motorcycles.

Table 3. Results of Classification of Training, Validation and Test Sets

Legend: TP-True Positive; TN-True Negative; Acc-Accuracy

\begin{tabular}{|c|c|c|c|c|c|c|c|c|c|c|c|}
\hline \multicolumn{3}{|c|}{ No. of samples } & \multicolumn{3}{c|}{ Combined results of classification for training, validation and test samples (Total of 500} \\
\hline \multicolumn{3}{|c|}{} & \multicolumn{3}{|c|}{ Training } & \multicolumn{3}{c|}{ Validation } & \multicolumn{3}{c|}{ Testing } \\
\hline Train & Valid & Test & TP & TN & Acc & TP & TN & Acc & TP & TN & Acc \\
\hline 150 & 175 & 175 & 67 & 74 & 0.9400 & 77 & 79 & 0.8914 & 76 & 78 & 0.8800 \\
\hline 175 & 150 & 175 & 80 & 82 & 0.9257 & 67 & 69 & 0.9067 & 73 & 82 & 0.8857 \\
\hline 175 & 175 & 150 & 71 & 81 & 0.8686 & 71 & 81 & 0.8686 & 60 & 67 & 0.8467 \\
\hline 200 & 125 & 175 & 82 & 88 & 0.8500 & 43 & 52 & 0.7600 & 65 & 77 & 0.8114 \\
\hline 200 & 150 & 150 & 80 & 90 & 0.8500 & 54 & 65 & 0.7933 & 51 & 64 & 0.7667 \\
\hline 200 & 175 & 125 & 84 & 95 & 0.8950 & 69 & 82 & 0.8629 & 42 & 57 & 0.7920 \\
\hline 225 & 100 & 175 & 97 & 103 & 0.8889 & 36 & 41 & 0.7700 & 72 & 77 & 0.8514 \\
\hline 225 & 125 & 150 & 93 & 102 & 0.8667 & 43 & 53 & 0.7680 & 62 & 64 & 0.8400 \\
\hline 225 & 150 & 125 & 95 & 104 & 0.8844 & 56 & 67 & 0.8200 & 43 & 55 & 0.7840 \\
\hline 225 & 175 & 100 & 105 & 107 & 0.9422 & 64 & 83 & 0.8400 & 41 & 45 & 0.8600 \\
\hline 250 & 100 & 150 & 106 & 116 & 0.8880 & 31 & 41 & 0.7200 & 55 & 64 & 0.7933 \\
\hline 250 & 125 & 125 & 103 & 111 & 0.8560 & 41 & 52 & 0.7440 & 40 & 59 & 0.7920 \\
\hline 250 & 150 & 100 & 108 & 118 & 0.9040 & 58 & 66 & 0.8267 & 33 & 40 & 0.7300 \\
\hline 275 & 100 & 125 & 120 & 120 & 0.8727 & 34 & 37 & 0.7100 & 45 & 50 & 0.7600 \\
\hline 275 & 125 & 100 & 129 & 127 & 0.9309 & 54 & 57 & 0.8880 & 44 & 42 & 0.8600 \\
\hline 300 & 100 & 100 & 126 & 143 & 0.8967 & 34 & 43 & 0.7700 & 35 & 43 & 0.7800 \\
\hline
\end{tabular}


Further, the partitioning of dataset into training, validation and testing sets has affects the classification accuracy. The classification performance depends on the size of the data sets and hence the results are summarized in Table 4.

Table 4. Impact of the Size of the Database on the Classification Performance

\begin{tabular}{|c|c|c|c|c|c|c|c|c|c|c|}
\hline $\begin{array}{c}\text { Classification } \\
\text { accuracy }\end{array}$ & \multicolumn{10}{|c|}{ Size of the database } \\
\hline $\begin{array}{c}\text { Size of the } \\
\text { database }\end{array}$ & $\mathrm{N}=100$ & $\mathrm{~N}=200$ & $\mathrm{~N}=300$ & \multicolumn{2}{c|}{$\mathrm{N}=400$} & \multicolumn{2}{c|}{$\mathrm{N}=500$} \\
\hline Condition & Healthy & Faulty & Healthy & Faulty & Healthy & Faulty & Healthy & Faulty & Healthy & Faulty \\
\hline Minimum & 98 & 96 & 90 & 94 & 88.7 & 82 & 89 & 88.5 & 68.4 & 82.8 \\
\hline Maximum & 100 & 100 & 100 & 98 & 96 & 95.3 & 97 & 97.5 & 90.8 & 94.8 \\
\hline Average & 99.96 & 99.47 & 98.33 & 97.10 & 92.90 & 92.47 & 93.26 & 93.07 & 78.66 & 89.15 \\
\hline
\end{tabular}

The average classification accuracy is over $97 \%$ for the databases of size $\mathrm{N}=100$ to $\mathrm{N}=200$ Hence the approach is reliable for a service station, where around 150 vehicles are serviced a day.

The samples recorded in real-world environments have an SNR of about 4.9. The experiment is extended for signals with different SNRs. Signals with SNR ranging from $0 \mathrm{~dB}$ to $10 \mathrm{~dB}$, are used for training and testing. The results of testing with different SNR are summarized in Table 5. Training and testing are carried out over the signals with the same SNR values.

Table 5. Classification Accuracy for Different SNRs

\begin{tabular}{|c|c|c|c|c|c|}
\hline $\begin{array}{c}\text { SNR } \\
\text { in dB }\end{array}$ & $\begin{array}{c}\text { True } \\
\text { Positive }\end{array}$ & $\begin{array}{c}\text { False } \\
\text { Negative }\end{array}$ & $\begin{array}{c}\text { True } \\
\text { Negative }\end{array}$ & $\begin{array}{c}\text { False } \\
\text { Positive }\end{array}$ & Accuracy \\
\hline $0 \mathrm{~dB}$ & 163 & 87 & 127 & 83 & 0.5800 \\
\hline $1 \mathrm{~dB}$ & 159 & 91 & 138 & 112 & 0.5940 \\
\hline $2 \mathrm{~dB}$ & 177 & 73 & 137 & 113 & 0.6280 \\
\hline $3 \mathrm{~dB}$ & 166 & 84 & 165 & 85 & 0.6620 \\
\hline $4 \mathrm{~dB}$ & 166 & 84 & 166 & 84 & 0.6640 \\
\hline $5 \mathrm{~dB}$ & 184 & 66 & 188 & 73 & 0.7440 \\
\hline $6 \mathrm{~dB}$ & 195 & 55 & 187 & 63 & 0.7640 \\
\hline $7 \mathrm{~dB}$ & 202 & 28 & 197 & 53 & 0.7980 \\
\hline $8 \mathrm{~dB}$ & 218 & 32 & 196 & 54 & 0.8280 \\
\hline $9 \mathrm{~dB}$ & 222 & 28 & 211 & 39 & 0.8660 \\
\hline $10 \mathrm{~dB}$ & 228 & 22 & 204 & 46 & 0.8640 \\
\hline
\end{tabular}

Naturally, the classification accuracy increases with increase in SNR of the signals. But the results are over $76 \%$ for signals with SNR of 6 and more. Hence the strength of the approach and the choice of the classifier are justified. 


\section{Conclusion}

The work classifies the motorcycles into healthy and faulty based on the slopes of the estimated pseudospectral regions. The samples are drawn from sound signals of healthy and faulty motorcycles of different makes and models. The results are consistent, with average classification accuracy over $78.66 \%$ in case of healthy and $89.15 \%$ in case of faulty motorcycles. Since it requires considerable amount of time for estimating the pseudospectrum and training the ANN, the method is not suitable for on-ride fault diagnosis. The work finds many applications including fault diagnosis of machines, musical instruments and diagnosing human diseases based on the sound.

\section{Acknowledgement}

We the authors thank the expert mechanics of Bagalkot City (Karnataka State, India) for sharing their fault diagnostic expertise and support for sound signal recording. We also thank Prof. Ramesh S. Wadawadagi for language corrections.

\section{Annexure 1. Details of the Healthy and Faulty Motorcycle Samples}

\begin{tabular}{|r|l|l|l|l|l|l|}
\hline Sl. No. & Model & $\begin{array}{l}\text { Kilom } \\
\text { eters } \\
\text { run }\end{array}$ & Age & Condition & $\begin{array}{l}\text { Recording } \\
\text { duration }\end{array}$ & $\begin{array}{l}\text { No. of } \\
\text { samples }\end{array}$ \\
\hline 1. & HH Spl + & 90991 & $11 \mathrm{Y}$ & Cylinder kit & $45 \mathrm{~s}$ & 28 \\
\hline 2. & Bajaj XCD125 & 14754 & $2 \mathrm{Y}$ & Faulty crank & $48 \mathrm{~s}$ & 30 \\
\hline 3. & HH Passion + & 1599 & $9 \mathrm{M}$ & Healthy & $45 \mathrm{~s}$ & 32 \\
\hline 4. & HH Spl + & 2385 & $1 \mathrm{Y}$ & Healthy & $53 \mathrm{~s}$ & 35 \\
\hline 5. & HH Spl Pro & 2142 & $4 \mathrm{M}$ & Healthy & $44 \mathrm{~s}$ & 34 \\
\hline 6. & HH CBZ Ex & 5727 & $1 \mathrm{Y}$ & Healthy & $46 \mathrm{~s}$ & 32 \\
\hline 7. & Bajaj CT 100 & 5116 & $1 \mathrm{Y}$ & Healthy & $51 \mathrm{~s}$ & 33 \\
\hline 8. & Pulser DTSI & 5010 & $10 \mathrm{M}$ & Healthy & $49 \mathrm{~s}$ & 34 \\
\hline 9. & HH CD100 & 62979 & $9 \mathrm{Y}$ & $\begin{array}{l}\text { Muffler } \\
\text { leakage }\end{array}$ & $54 \mathrm{~s}$ & 32 \\
\hline 10. & HH Super Spl & 54777 & $5 \mathrm{Y}$ & $\begin{array}{l}\text { Muffler } \\
\text { leakage }\end{array}$ & $47 \mathrm{~s}$ & 31 \\
\hline 11. & HH Passion & 74737 & $6 \mathrm{Y}$ & $\begin{array}{l}\text { Silencer } \\
\text { leakage }\end{array}$ & $49 \mathrm{~s}$ & 30 \\
\hline 12. & HH Passion + & 61,999 & $9.5 \mathrm{Y}$ & Timing chain & $50 \mathrm{~s}$ & 28 \\
\hline 13. & HH CD Dawn & 73888 & $6 \mathrm{Y}$ & Timing chain & $48 \mathrm{~s}$ & 30 \\
\hline 14. & HH CBZ & 81014 & $8 \mathrm{Y}$ & Timing chain & $64 \mathrm{~s}$ & 31 \\
\hline 15. & HH Spl + & 15711 & $2 \mathrm{Y}$ & Valve setting & $51 \mathrm{~s}$ & 29 \\
\hline 16. & HH Splend & 50632 & $5 \mathrm{Y}$ & Valve setting & $58 \mathrm{~s}$ & 31 \\
\hline Total No. of samples & & & & & 500 \\
\hline & & & & & \\
\hline
\end{tabular}




\section{References}

[1] http://www.moneylife.in/article/car-sales-in-india-likely-to-expand-3-5-in-fy14-siam/32142.html (Predictions of SIAM for two-wheeler sales in financial year 2013-14]).

[2] B. S. Anami, V. B. Pagi and S. M. Magi, "Wavelet Based Acoustic Analysis for Determining Health Condition of Two-Wheelers”, Elsevier J. Applied Acoustics, vol. 72, no. 7, (2011), pp. 464-469.

[3] Z. JunHong and H. Bing, “Analysis of Engine Front Noise using Sound Intensity Techniques”, Mechanical Systems and Signal Processing, vol. 19, (2005), pp. 213-221.

[4] J. D. Wu, E. C. Chang, S. Y. Liao, J. M. Kuo and C. K. Huang, "Fault Classifica, tion of a Scooter Engine Platform using Wavelet Transform and Artificial Neural Network”, Proceedings of the International MultiConference of Engineers and Computer Scientists, IMECS 2009, Hong Kong, vol. 1, (2009) March 1820, pp. 58-63.

[5] W. Liao, P. Han and X. Liu, "Fault Diagnosis for Engine Based on EMD and Wavelet Packet BP Neural Network", Proceedings of the Third International Symposium on Intelligent Information Technology Application, (2009), pp. 672-676.

[6] K. Heidarbeigi, H. Ahmadi, M. Omid and A. Tabatabaeefar, "Fault Diagnosis of Massey Ferguson Gearbox Using Power Spectral Density”, J. Agricultural Technology, vol. 5, no. 1, (2009), pp. 1-6.

[7] J. Lin and M. J. Zuo, "Gearbox Fault Diagnosis using Adaptive Wavelet Filter, Mechanical Systems and Signal Processing”, vol. 17, no. 6, (2003), pp. 1259-1269.

[8] J. Cheng, D. Yu and Y. Yang, “A Fault Diagnosis Approach for Gears Based on IMF ARModel and SVM”, EURASIP J. Advances in Signal Processing, Article ID 647135, doi:10.1155/2008/647135, (2008).

[9] S. Yella, N. Gupta and M. Dougherty, “Condition Monitoring Using Pattern Recognition Techniques on Data from Acoustic Emissions”, Proceedings of the 5th International Conference on Machine Learning and Applications (ICMLA'06), Orlando, Florida, (2006) December 14-16, pp. 3-9.

[10] C. J. Verucchi, G. G. Acosta and F. A. Benger, “A Review on Fault Diagnosis of Induction Machines, vol. 38, (2008), pp. 113-121.

[11] A. Datta, C. Mavroidis, J. Krishnasamy and M. Hosek, "Neural Network based Fault Diagnostics of Industrial Robots using Wavelet Multi-resolution Analysis”, Proceedings of the American Control Conference, New York City, USA, (2007) July 11-13, pp. 1858-1863.

[12] V. Belotti, F. Crenna, R. C. Michelini and G. B. Rossi, "Wavelet Signal Processing Applied to Railway Wheelflat Detection”, Proceedings of the XVII IMEKO World Congress Metrology in the 3rd Millennium Dubrovnik, Croatia, (2003) June 22-27, pp. 1434-1437.

[13] B. S. Anami and V. B. Pagi, "An Acoustic Signature Based Neural Network Model for Type Recognition of Two-Wheelers”, Proceedings of the IEEE International Conference on Multimedia Systems, Signal Processing and Communication Technologies, Aligarh, (2009) March 14-16, pp. 28-31.

[14] M. Ayoubi, "Fuzzy Systems Design Based on a Hybrid Neural Structure and Application to the Fault Diagnosis of Technical Processes”, Control Engg., Practice, vol. 4, no. 1, (1996), pp. 35-42.

[15] J. Lin, "Feature Extraction of Machine Sound using Wavelet And Its Application in Fault Diagnosis", NDT\&E International, vol. 34, (2001), pp. 25-30.

[16] X. Wang and H. Qi, “Acoustic target classification using distributed sensor arrays”, IEEE Int. Conf. on Acoustics, Speech, and Signal Processing (ICASSP), Orlando, FL, USA, (2002) May13-17, pp. IV-4186-IV4186.

[17] J. E. Lopez, H. Han Chen and J. Saulnier, "Target Identification Using Wavelet-based Feature Extraction and Neural Network Classifiers”, Tech. rep., CYTEL Systems, Inc., (1999).

[18] S. N. Engin and K. Gulez, "A Wavelet Transform - Artificial Neural Networks (WT-ANN) based Rotating Machinery Fault Diagnostics Methodology”, Proceedings of the IEEE NSIP’ 99, Falez Hotel, Antalya, Turkey, (1999), pp. 714-720.

[19] H. Maciejewski, J. Mazurkiewicz, K. Skowron and T. Walkowiak, "Neural Networks for Vehicle Recognition”, Proceedings of the 6th International conference on Microelectronics for Neural Networks, Evolutionary and fuzzy Systems, (1997), pp. 292-296.

[20] A. Averbuch, V. A. Zheludev, N. Rabin and A. Schclar, "Wavelet-based acoustic detection of moving vehicles”, Multidimensional Systems and Signal Processing, vol. 20, no. 1, (2009), pp. 1-25.

[21] K. Mehrotra, C. K. Mohan and S. Ranka, "Elements of Artificial Neural Networks”, The MIT Press, (1996).

[22] S. Xu and L. Chen, "A Novel Approach for Determining the Optimal Number of Hidden Layer Neurons for FNN's and Its Application in Data Mining”, 5th International Conference on Information Technology and Applications, Queensland, (2008), pp. 683-686.

[23] http://www.mathworks.in/help/toolbox/signal/pmusic.html. 